\title{
Il labirinto delle finanze: un gioco sull'educazione finanziaria per le scuole secondarie di secondo grado
}

\section{The maze of finance: a financial literacy game for upper secondary schools}

\author{
Giulia Bernardi ${ }^{\star}$ Elena Brambilla ${ }^{\circ}$ e Paola Landra \\ *Dipartimento formazione e apprendimento - SUPSI, Locarno, Svizzera \\ ${ }^{\circ}$ Liceo Scientifico Statale Vittorio Veneto - Milano, Italia \\ ${ }^{\star}$ Istituto Professionale di Stato L. Milani - Meda, Italia
}

Sunto / L'educazione finanziaria è un argomento di sempre maggior interesse al giorno d'oggi, in particolare per la formazione degli studenti delle scuole secondarie, in modo che diventino soggetti attivi e consapevoli nel prendere decisioni in ambito economico e finanziario. All'interno del progetto Edufin@Polimi è stato creato un gioco da proporre nelle scuole secondarie di secondo grado per avvicinare i ragazzi ai concetti principali di matematica finanziaria, come le leggi di capitalizzazione, il rendimento degli investimenti, le condizioni dei prestiti. In questo articolo viene descritta la fase di progettazione del gioco e si riportano i risultati delle prime sperimentazioni realizzate in tre diverse scuole.

Parole chiave: educazione finanziaria; caccia al tesoro; gamification.
Abstract / Nowadays financial literacy is one of the topics in school's curricula that is attracting more attention; a special focus is given to programs for secondary school students that should become active and informed decision makers in the economic and financial setting. Within the project Edufin@Polimi, a game was developed to let students discover some of the main ideas of financial mathematics, such as simple and compound interest, investment, loans rules. In this article we describe the design of this game and report the results of the first experimentations realized in three different upper secondary schools.

Keywords: financial literacy; treasure hunt; gamification.

Negli ultimi anni, il tema delle conoscenze e abilità finanziarie dei cittadini è diventato un argomento rilevante all'interno del dibattito pubblico, soprattutto a fronte delle crisi economiche delle banche nel 2008 e della nascita di nuovi strumenti finanziari di sempre maggiore complessità.

A conferma della crescente importanza di questo tema, già dal 2012 l'OCSE ha inserito in alcune delle nazioni coinvolte nelle rilevazioni PISA I'ambito financial literacy (OECD, 2014).

L'alfabetizzazione finanziaria o financial literacy è definita come

«il processo per mezzo del quale i consumatori di prodotti finanziari migliorano la loro comprensione su prodotti, concetti e rischi finanziari e, attraverso l'informazione, l'istruzione e consigli oggettivi, sviluppano le capacità e la fiducia 
nella propria consapevolezza dei rischi e delle opportunità finanziarie, di sapere dove chiedere aiuto, e intraprendere altre azioni efficaci per migliorare il proprio benessere economico».

(INVALSI, 2017, p. 5)

L'obiettivo dell'educazione finanziaria non è quindi solo la trasmissione dei concetti, ma anche la promozione dell'abilità di prendere decisioni consapevoli in ambito finanziario ed economico (Lusardi, 2015) per migliorare il benessere finanziario, portando benefici non solo ai singoli individui ma anche alla società, in modo che i giovani si sentano in grado di partecipare attivamente alla vita economica.

Una scarsa conoscenza dei temi legati agli aspetti economici e finanziari rende gli studenti, e quindi i futuri cittadini, soggetti poco consapevoli nel prendere decisioni in questo ambito e, dunque, più esposti ai rischi (Lusardi 2012). Alcuni studi (Lusardi, 2015; INVALSI, 2017), hanno indicato quanto siano rilevanti nei risultati dei test su questi argomenti anche fattori non cognitivi, ma dipendenti dal contesto socioeconomico e familiare. Ad esempio, molte delle conoscenze finanziarie dei giovani vengono acquisite soprattutto dalle conversazioni con genitori e amici. Per questo motivo Lusardi, Mitchell e Curto (2010) sottolineano quanto sia importante inserire programmi di educazione finanziaria in tutte le scuole, per poter raggiungere anche quegli studenti che sono più svantaggiati perché non frequentano abitualmente persone adulte con conoscenze in ambito finanziario, e come sia meglio offrire programmi di educazione finanziaria durante il periodo scolastico, prima che gli individui si trovino ad essere coinvolti nell'assunzione di responsabilità di carattere finanziario, quali la sottoscrizione di prestiti, mutui e contratti di vario tipo.

Le caratteristiche dei programmi di educazione finanziaria fanno la differenza sui risultati ottenuti: Kaiser e Menkhoff (2017) notano che un effetto positivo si ha quando l'educazione finanziaria avviene in un "teachable moment", cioè quando l'insegnamento è associato a una situazione in cui si devono prendere decisioni di immediata rilevanza per il gruppo.

A livello internazionale, le iniziative da parte delle istituzioni per far fronte a questa necessità sono state diverse. Ad esempio, nel 2016 in Svizzera è stata fondata l'Associazione FinanceMission ${ }^{1}$ da parte di due associazioni di insegnanti (LCH, Dachverband Lehrerinnen und Lehrer Schweiz e SER, Syndicat des Enseignants Romands) e dall'Unione delle Banche Cantonali Svizzere (UBCS); in Italia nel 2017 è stato fondato su decreto del Ministro dell'economia e delle finanze, di concerto con il Ministro dell'istruzione, dell'università e della ricerca e con quello dello sviluppo economico, il Comitato per l'Educazione Finanziaria ${ }^{2}$ con lo scopo di coordinare le diverse iniziative volte a promuovere l'educazione finanziaria tra la popolazione.

In questo filone di iniziative è nato anche il progetto Edufin@Polimi, coordinato dal gruppo di ricerca in Finanza Qualitativa del Dipartimento di Matematica del Politecnico di Milano, che ha come scopo la diffusione di progetti di educazione finanziaria, con una particolare attenzione agli studenti delle scuole secondarie di secondo grado $^{3}$. Le proposte sviluppate all'interno di questo progetto sono varie: un sito con alcune pagine dedicate a temi specifici del mondo della finanza (https://www.

1. Il sito del progetto è https://financemission.ch/?lang=it.

2. Il sito del comitato è http://www.quellocheconta.gov.it/it/chi-siamo/comitato/.

3. La scuola secondaria di secondo grado in Italia dura cinque anni e corrisponde all'ultimo anno di scuola media e alla scuola media superiore o alle scuole professionali nel Canton Ticino. 
imparalafinanza.it), un percorso didattico per permettere ai docenti di matematica di introdurre alcuni argomenti di matematica finanziaria utilizzando modalità didattiche innovative, come la flipped classroom (Bergmann \& Sams, 2012), e un gioco, sotto forma di caccia al tesoro, che consenta di introdurre questi temi facendo avvicinare gli studenti al mondo della finanza con un approccio ludico e informale. La prima caccia al tesoro a tema finanziario è stata realizzata nell'ottobre 2017, mese dedicato all'educazione finanziaria presso il Politecnico di Milano, e ha coinvolto una cinquantina di studenti dell'ultimo anno di alcuni licei classici. A partire da questa esperienza, ripetuta l'anno successivo, è stato avviato un progetto più ampio per realizzare "il labirinto delle finanze", un gioco sui temi dell'educazione finanziaria da proporre nelle scuole secondarie di secondo grado del territorio, che fosse realizzabile autonomamente dai docenti interessati, e che si potesse rivolgere a tutte le classi, di qualsiasi indirizzo scolastico.

In questo articolo descriviamo i primi mesi di questa esperienza di creazione e realizzazione del gioco "il labirinto delle finanze", in particolare la fase di progettazione che ha previsto la collaborazione tra ricercatori e insegnanti delle scuole secondarie di secondo grado e le prime sperimentazioni realizzate nella primavera del 2019 in alcune classi di tre diversi istituti.

\section{Il gioco "il labirinto delle finanze"}

Da un primo momento esplorativo di confronto tra ricercatori e insegnanti, è emersa in primo luogo la difficoltà da parte dei docenti nello stabilire come affrontare con i propri studenti i temi legati alla matematica finanziaria. Effettivamente si tratta di argomenti complessi, per i quali spesso il docente non possiede la preparazione specifica necessaria e che sono difficili da inserire in una programmazione annuale già densa. Per strutturare la nostra proposta abbiamo scelto di seguire l'esempio di Aprea, Schultheis e Stolle (2018), che suggerisce di sfruttare un approccio ludico, vicino al mondo della gamification ${ }^{4}$, interessante per le seguenti ragioni:

- introduce una variazione nella routine scolastica, che gli alunni vivono in particolari momenti come pesante e oppressiva;

- concentra in un lasso di tempo ben definito e contenuto le ore destinate allo sviluppo dell'argomento specifico (matematica finanziaria);

- il gioco, organizzato in piccole squadre, permette di mescolare i compagni di classe, e con essi le abilità, le competenze, le conoscenze, le preferenze, creando spazio per lasciare emergere talenti non visibili durante le ore di lezione e sensibilità e tratti umani tenuti nascosti;

- il gioco digitale promuove il divertimento, motiva e stimola un atteggiamento positivo verso la materia da apprendere.

A partire da queste considerazioni è stato realizzato un gioco a squadre, in cui gli studenti potessero avvicinarsi ai temi della matematica finanziaria. Per quanto riguarda la progettazione del gioco, si sono presi in considerazione diversi aspetti: la

4. Il termine gamification deriva dall'inglese game (gioco) e indica I'utilizzo di elementi tipici del gioco (come punti da ottenere, competizione con gli altri, regole da seguire) in contesti non ludici. 
necessità di correggere velocemente le risposte inserite, la possibilità di adattare il gioco a scuole con spazi e ambienti diversi, la possibilità di coinvolgere anche docenti non di matematica per la supervisione del gioco (richiesta fatta dai docenti di matematica stessi, soprattutto nel caso di classi numerose, per poter svolgere il gioco in spazi diversi ma avere sempre un unico docente supervisore). Nel seguito di questo paragrafo spiegheremo le scelte che abbiamo compiuto entrando nel dettaglio della descrizione del gioco, delle modalità di svolgimento e degli argomenti di matematica finanziaria che sono trattati.

\subsection{La struttura del gioco}

Il gioco è strutturato in quattro tappe di cui una doppia, in modo che ad ogni tappa venga affrontato un argomento diverso (vedi Tabella 1). Per poter rendere il gioco replicabile facilmente in ogni scuola, indipendentemente dagli spazi a disposizione, si sono stabiliti a priori i nomi delle tappe relativi a luoghi: Aula, Banca, Casa, Negozio. Quando viene organizzato il gioco in una scuola, il docente deve associare queste tappe ai luoghi reali che ha a disposizione per lo svolgimento del gioco che assumono metaforicamente tali significati; ad esempio, la palestra può diventare la "Banca", la biblioteca il "Negozio" ecc. Per sapere quali sono gli spazi a disposizione dello svolgimento del gioco, all'inizio dell'attività ogni squadra riceve una cartina della propria scuola in cui alcuni luoghi sono indicati con i nomi che indicano le possibili tappe. Per rendere più complesso il gioco, oltre alle quattro tappe che realmente devono essere visitate dai giocatori durante la caccia al tesoro, abbiamo previsto anche l'inserimento di "tappe finte" con la funzione di distrattori (Agenzia, Edicola, Parco, Ristorante) che nel corretto svolgimento del gioco non dovrebbero essere raggiunte dalle squadre.

A ogni tappa è prevista la presenza di un docente responsabile il cui compito è di accogliere le squadre e distribuire il materiale. Ogni squadra riceve quindi, dopo essere arrivata nel luogo corretto, un documento con alcune spiegazioni che dovrebbero permettere di acquisire le conoscenze e le informazioni necessarie per rispondere alle domande della tappa. Le spiegazioni e le informazioni per rispondere alle domande sono presentate con diverse modalità: paragrafi di testi, grafici, tabelle, brevi video da guardare con il proprio smartphone. Al fondo di ogni scheda di materiali è presente un riquadro con un QR Code da cui è possibile accedere al formulario online che contiene le domande relative alla tappa e in cui i giocatori possono inserire le risposte (Figura 1).

Figura 1

Un esempio di materiale ricevuto in una tappa. Sul foglio ci sono alcune spiegazioni espresse in forma testuale e tramite un video accessibile con il QR Code. II QR Code in fondo alla pagina permette di accedere alle domande del formulario alle quali occorre rispondere.

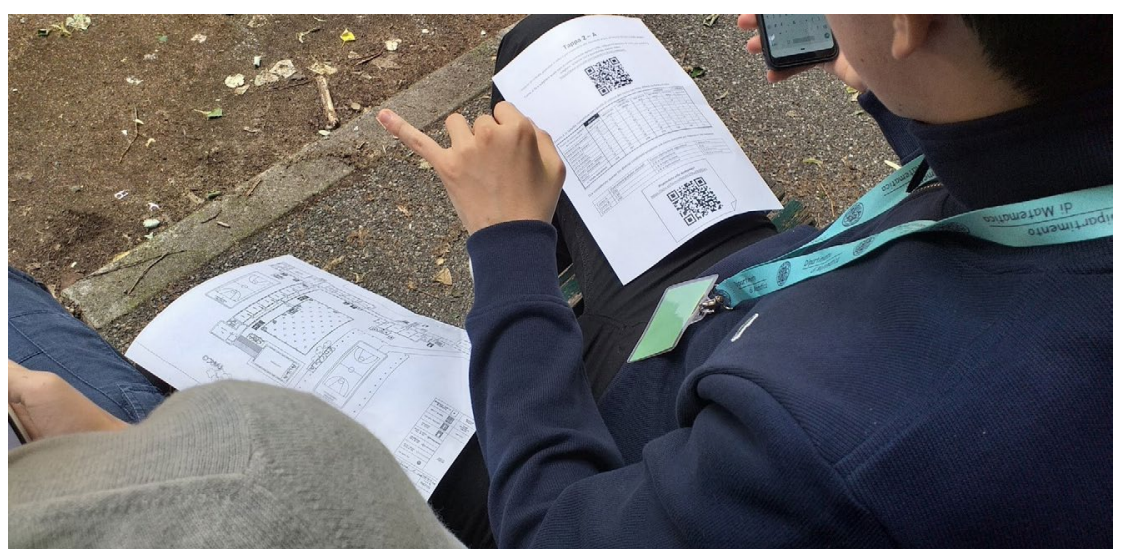


Ogni squadra deve inserire le risposte nel formulario online, che è stato creato utilizzando Google Forms ${ }^{5}$. Nel nostro gioco le domande sono a risposta multipla o a risposta aperta univoca (nei casi in cui la risposta è un dato numerico); ad ogni domanda è assegnato un punto, in modo che il punteggio massimo per ogni tappa corrisponda al numero di domande. La struttura del modulo creato con Google Forms permette ai giocatori di lavorare in autonomia nell'inserimento delle risposte, di vedere in modo automatico il punteggio ottenuto al termine dell'inserimento e di verificare quali sono le risposte sbagliate.

Prima di rispondere alle domande specifiche di una tappa, ogni squadra deve inserire il suo nome, scelto all'inizio del gioco, e il numero del tentativo, per indicare quante volte la squadra ha già provato a rispondere alle domande. Nel regolamento del gioco abbiamo previsto che una squadra non possa passare alla tappa successiva finché non abbia realizzato il punteggio massimo, così da portare gli studenti a rivedere i propri errori, a ritornare sui vari concetti esposti fino ad individuare le risposte corrette. Questo sistema di correzione automatica rende gli studenti più autonomi e indipendenti nel processo di autocorrezione, oltre a permettere di avere anche docenti di diverse materie come supervisori delle tappe e a velocizzare il processo di correzione.

Una volta realizzato il punteggio massimo, il capitano della squadra lo mostra al docente responsabile della tappa che consegna un indizio per raggiungere la tappa successiva.

Visto che è il docente stesso a consegnare l'indizio successivo, esiste la possibilità di modificare la regola per cui occorre raggiungere il punteggio massimo per accedere alla tappa successiva, stabilendo invece un punteggio minore da raggiungere. Questa modifica può essere gestita facilmente dal responsabile della tappa nel momento in cui distribuisce il materiale, con il semplice avviso di quale sia il punteggio da ottenere per proseguire il gioco. I docenti possono fare questa variazione, per velocizzare il gioco in caso di rallentamenti alle tappe precedenti o per alleggerire le ultime tappe in caso di necessità, ad esempio per calo dell'attenzione o della motivazione da parte degli studenti verso le ultime fasi del gioco.

L'indizio che le squadre ricevono per poter raggiungere la tappa successiva è di natura non finanziaria: un indovinello, un anagramma o un messaggio cifrato; è stata fatta questa scelta per rendere il gioco ancora più coinvolgente e inserire anche degli elementi puramente ludici (vedi Allegato 1). La risposta di ogni indizio fornisce il nome della tappa successiva. Ad esempio, dopo aver completato la tappa "Casa" le squadre ricevevano questo indizio:

Questo anagramma dovrete dedurre, per sapere dove il gioco vi vuole condurre:

AZIONATE INDEGNO

La soluzione dell'anagramma, con l'indicazione della tappa successiva, è "Andate in negozio".

5. Abbiamo scelto questo strumento perché presenta diversi vantaggi, permette infatti di creare diversi tipi di domanda (a risposta multipla, aperte, con griglie ecc.); inserire automaticamente la risposta corretta; assegnare dei punteggi rispetto alle risposte; accedere facilmente ai questionari tramite un link senza che sia necessario registrarsi; salvare automaticamente tutte le risposte in tempo reale. 


\subsection{Gli argomenti}

La progettazione del gioco è avvenuta soprattutto a partire dal materiale presente nel MOOC ${ }^{6}$ "Finanza per tutti" creato dal Dipartimento di Matematica del Politecnico di Milano. In questo corso online, rivolto alla cittadinanza, sono affrontati diversi temi di matematica finanziaria in modo accessibile anche per un pubblico di non esperti. La prima settimana del MOOC è dedicata ai concetti di base: il conto corrente, le leggi di capitalizzazione, i tassi di interesse di azioni e obbligazioni, i costi di prestiti, finanziamenti e mutui. La seconda settimana invece riguarda in modo più specifico i rischi collegati agli investimenti, affrontando temi come la diversificazione, la psicologia delle scelte di investimento, le assicurazioni e le misure di rischio. I temi affrontati nell'ultima settimana di corso invece riguardano il risparmio, in particolare i prodotti offerti da banche e istituzioni, per cui si parla di profilatura del rischio, derivati, fondi comuni di investimento, fondi pensione.

A partire da questo materiale abbiamo deciso di selezionare i temi che potessero essere più vicini alla realtà degli studenti di scuola superiore e per cui ci fossero degli argomenti rilevanti anche dal punto di vista matematico. Nella Tabella 1 abbiamo riassunto gli argomenti presenti ad ogni tappa del gioco.

\begin{tabular}{|l|l|l|}
$\begin{array}{l}\text { Nome } \\
\text { della tappa }\end{array}$ & Argomento & Contenuti \\
\hline$\# 1$ - Aula & Ripasso & $\begin{array}{l}\text { Scrivere rappresentazioni equivalenti dei numeri } \\
\text { in forma percentuale, frazionaria o decimale. } \\
\text { Leggere e interpretare grafici. }\end{array}$ \\
\hline \#2A - Banca 1 & Conto corrente & $\begin{array}{l}\text { Calcolare il costo del conto corrente rispetto } \\
\text { ad alcune offerte e alcune tipologie di utilizzo. }\end{array}$ \\
\hline$\#$ 2B - Banca 2 & $\begin{array}{l}\text { Leggi di } \\
\text { capitalizzazione }\end{array}$ & $\begin{array}{l}\text { Utilizzare le leggi di capitalizzazione semplice e } \\
\text { composta per calcolare il montante con orizzonti } \\
\text { temporali di uno o più anni. }\end{array}$ \\
\hline$\#$ - Casa & Investimenti & $\begin{array}{l}\text { Valutare la rischiosità di un investimento e } \\
\text { calcolare il rendimento. }\end{array}$ \\
\hline Negozio & Prestiti & $\begin{array}{l}\text { Scegliere le condizioni di prestito più convenienti } \\
\text { e confrontare prestiti con tassi di interesse su } \\
\text { orizzonti temporali minori dell'anno. }\end{array}$ \\
\hline
\end{tabular}

\subsubsection{La prima tappa: "Aula"}

Volendo progettare un gioco adatto a tutte le classi e a tutti gli indirizzi, abbiamo cercato di far sì che i prerequisiti matematici fossero relativi ad argomenti già affrontati durante gli anni di scuola precedenti: percentuali, lettura dei grafici, utilizzo della calcolatrice, interpretazione di formule letterali, nozioni di base di probabilità.

Abbiamo deciso di inserire una prima tappa in cui le domande fossero relative a questi argomenti, in modo da permettere un ripasso generale dei concetti di base e sottolineare gli aspetti matematici da tenere più in considerazione nel resto del gio-

6. La sigla MOOC sta per Massive Open Online Course e indica un corso online gratuito accessibile a tutti. Il corso di cui si parla in questo articolo è accessibile dalla piattaforma https://www.pok.polimi.it/. 
co. Le domande presenti in questa prima tappa (vedi Allegato 2) sono otto a risposta chiusa, relative appunto al calcolo delle percentuali, all'inversione di una formula e alla lettura di un grafico in cui due linee spezzate rappresentano l'andamento dei costi di due biglietti aerei nel corso di alcuni mesi.

\subsubsection{La seconda tappa: "Banca"}

La seconda tappa è una tappa doppia in cui vengono affrontati due argomenti diversi: i costi dei conti correnti e le leggi di capitalizzazione.

In particolare, la tappa 2A è dedicata a stabilire il costo di gestione di un conto corrente considerando le spese fisse e le spese variabili, che dipendono dal numero di operazioni effettuate. Tra il materiale a disposizione degli studenti è stato inserito un breve video in cui viene spiegato che cos'è l'indicatore sintetico di costo (ISC) ${ }^{7}$ di un conto corrente e viene fornito un esempio di calcolo di questo indice. Le domande (vedi Allegato 3) chiedono di confrontare le condizioni di tre diversi conti correnti per individuare quale sia la più vantaggiosa a seconda del profilo dell'utente, quindi del numero di operazioni effettuate. Dal punto di vista matematico si tratta di risolvere alcune equazioni e disequazioni di primo grado, dato che il costo del conto corrente include un costo fisso annuale e un costo variabile dipendente dal numero di operazioni e può quindi essere rappresentato da funzioni lineari a tratti.

$\checkmark a$ considerato che per alcuni argomenti di matematica finanziaria sono necessarie conoscenze matematiche più avanzate, ma in questi casi abbiamo cercato di semplificare le domande per far sì che tali argomenti fossero ugualmente accessibili; scelta che è stata fatta ad esempio per quanto riguarda l'argomento delle leggi di capitalizzazione, trattate nella tappa 2B.

Le leggi di capitalizzazione permettono di calcolare il montante $(M)$, cioè il valore finale, di un investimento di denaro, solitamente indicato con $C$ e chiamato capitale iniziale, depositato per un periodo $t$ di tempo a un determinato tasso di interesse $i$. Le due leggi di capitalizzazione presentate in questo gioco sono quella di capitalizzazione semplice, in cui gli interessi vengono calcolati solo sul capitale iniziale, e quella di capitalizzazione composta, in cui gli interessi vengono calcolati ogni volta sul montante attuale, cioè non solo sul capitale iniziale ma anche sugli interessi già maturati fino a quel periodo.

Dato un capitale iniziale $C$ e un tasso di interesse annuale $i$, la formula per il calcolo del montante dopo $t$ anni con la legge di capitalizzazione semplice è

$$
M=C(1+i t)
$$

mentre con la legge di capitalizzazione composta è

$$
M=C(1+i)^{t} \text {. }
$$

Come si può notare, la legge di capitalizzazione semplice è una legge lineare, mentre quella di capitalizzazione composta è esponenziale, argomento che non viene trattato nel primo biennio nelle scuole secondarie di secondo grado italiane.

Per evitare che gli studenti si trovassero in difficoltà su aspetti matematici, nel filmato $^{8}$ in cui si parla di leggi di capitalizzazione, la formula è introdotta in modo ricorsivo, calcolando il montante per il primo anno con il tasso di interesse $i$ e poi calcolando il montante per il secondo anno applicando il tasso di interesse $i$ al nuovo

7. II video sull'indicatore sintetico di costo è visibile al link: https://wnw.youtube.com/watch?v=X15GcvDkR0A\& 8. Il video sulle leggi di capitalizzazione è visibile al link: https://www.youtube.com/watch?v=mf4RwxFJxK0. 
montante ecc. In questo modo non è necessario conoscere la funzione esponenziale, che anzi può essere introdotta proprio a partire da queste osservazioni, ma è sufficiente avere dimestichezza con il calcolo aritmetico di base. Le domande relative a questo argomento (vedi Allegato 4) chiedono di confrontare le due leggi di capitalizzazione calcolando il montante per un investimento iniziale di $100 €$, con un tasso annuale del $4 \%$ dopo un anno e dopo tre anni.

\subsubsection{La terza tappa "Casa"}

L'argomento della tappa successiva riguarda gli investimenti. II materiale a disposizione dei giocatori è una scheda (vedi Allegato 5) con alcuni grafici che rappresentano I'andamento di titoli azionari, un breve brano riguardante gli strumenti per misurare la rischiosità di un titolo e un altro paragrafo per introdurre un video ${ }^{9}$ sul tema dell'avversione o propensione al rischio delle persone. I due concetti fondamentali su cui è incentrata la tappa sono quelli di rendimento e volatilità.

Il rendimento si calcola rispetto ad un certo periodo di tempo ed è definito come il rapporto tra la variazione del prezzo del titolo tra l'inizio e la fine del periodo e il prezzo ad inizio periodo. La volatilità del titolo è la misura di quanto in media i rendimenti si allontanino dal rendimento medio, ovvero la deviazione standard dei rendimenti. Tanto più il rendimento si discosta dal suo valor medio, tanto più è elevata la volatilità e il titolo è considerato rischioso.

Il tema della valutazione del rischio di investimenti e prestiti è un tema molto complesso; abbiamo deciso comunque di mantenerlo in questa versione semplificata all'interno del gioco in quanto di grande attualità e perché coinvolge diversi aspetti rilevanti anche dal punto di vista matematico: interpretazione dei grafici, valutazioni probabilistiche e calcolo di indici statistici. Ad esempio, a partire dall'osservazione del grafico in Figura 2 viene chiesto di calcolare il rendimento di un'azione del titolo Appela tra il 2014 e il 2019 o in quale anno un'azione del titolo Samugu acquistata nel 2017 risulta redditizia. Per rispondere a queste domande gli studenti devono mobilitare diverse competenze: comprendere le spiegazioni presenti sulla scheda per estrapolare il significato di rendimento, tradurre tale concetto in linguaggio matematico, recuperare i dati numerici dal grafico ecc.

Figura 2

Uno dei grafici presenti nelle domande della terza tappa.

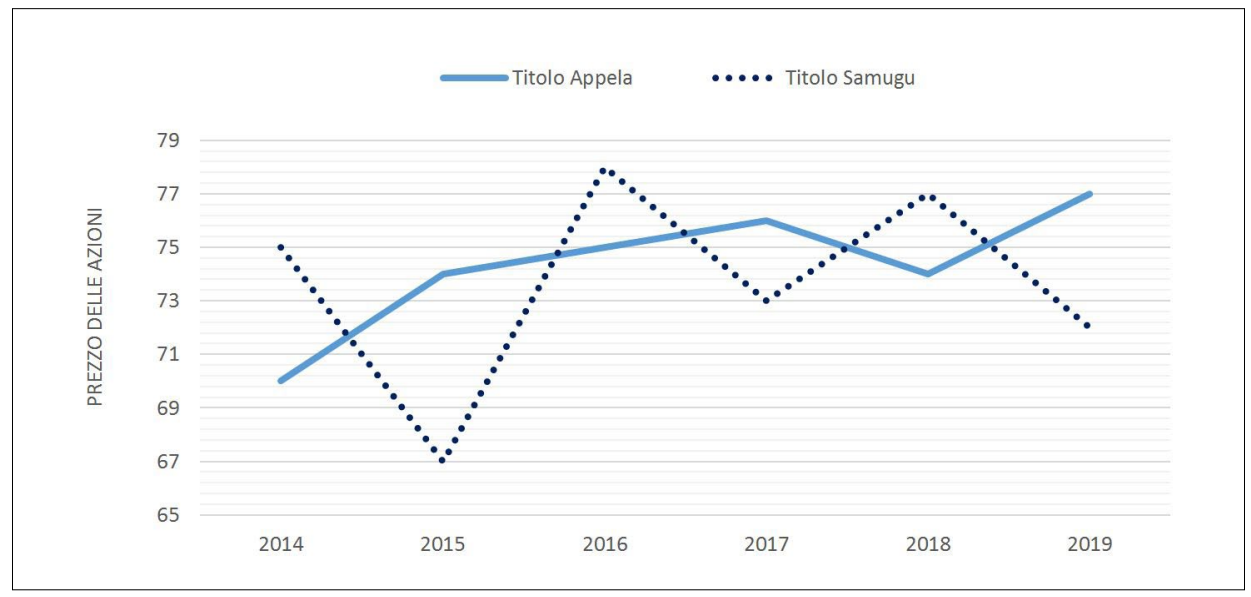

9. Il video sull'avversione al rischio è visibile al link: https://www.youtube.com/watch?v=kP4gSR_bgGo. 


\subsubsection{La quarta tappa: "Negozio"}

L'ultima tappa del gioco è dedicata al mondo dei prestiti, in particolare a due argomenti diversi: il significato delle sigle TAN e TAEG, presenti nelle pubblicità dei finanziamenti, e il confronto tra prestiti con orizzonti temporali diversi, con un accenno al tema dell'usura. Per quanto riguarda le condizioni generali dei prestiti, il tema non può ovviamente essere esaurito in una semplice tappa di gioco, ma abbiamo scelto di inserirlo almeno per porre I'attenzione degli studenti su questa tematica e fornire degli stimoli e dei materiali per un eventuale approfondimento. Dopo aver visto delle pubblicità di alcuni prestiti e avere a disposizione un link a una pagina web di approfondimento ${ }^{10}$, i giocatori devono rispondere ad alcune domande in cui viene chiesto di esplicitare le definizioni di queste sigle, di riflettere sulle condizioni offerte dalle tre pubblicità e confrontarle tra loro (vedi Allegato 6). II secondo argomento viene presentato attraverso un video ${ }^{11}$, in cui c'è un esempio di applicazione della legge di capitalizzazione composta con un tasso di interesse su un orizzonte temporale diverso dall'anno. Le domande riguardano proprio il calcolo del valore del prestito nel caso in cui i tassi di interesse siano il 4,5\% quadrimestrale o il $4 \%$ trimestrale.

\section{3 hasperianationo}

In questo paragrafo raccontiamo le osservazioni emerse durante le prime sperimentazioni del gioco, svolte tra aprile e maggio 2019 in tre diverse scuole secondarie di secondo grado (un liceo scientifico, un istituto professionale e un istituto tecnico commerciale) con sei classi coinvolte in totale. In particolare, hanno partecipato in quattro momenti diversi:

- una classe seconda di un liceo scientifico (20 studenti, divisi in 7 squadre);

- due classi seconde di un istituto professionale (31 studenti, divisi in 8 squadre);

- una classe seconda di un liceo scientifico (22 studenti, divisi in 8 squadre);

- una classe terza e una classe quarta di un istituto tecnico commerciale (40 studenti, divisi in 10 squadre).

Durante ogni sperimentazione si sono dovuti modulare in modo differente gli aspetti logistici e organizzativi, a seconda della disponibilità della scuola e dei docenti coinvolti. Ad esempio, quando il gioco è stato proposto con le classi del liceo scientifico sono state formate squadre da 2 o 3 giocatori, mentre negli altri casi le squadre erano costituite da 3 o 4 studenti. Le squadre sono sempre state formate tramite un sorteggio casuale, ma durante la sperimentazione con le classi dell'istituto commerciale sono state create in modo che metà dei componenti della squadra fosse di terza e metà fosse di quarta, visto che quest'ultima classe aveva già svolto alcune lezioni di matematica finanziaria e l'obiettivo era creare squadre in cui la conoscenza degli studenti su questi argomenti fosse più omogenea possibile.

Come metodologia utilizzata per tener traccia dell'esperienza, oltre all'osservazione da parte dei ricercatori, abbiamo raccolto le risposte fornite dagli studenti alle

10. La pagina web riguardante le sigle dei prestiti è https://www.imparalafinanza.it/TAN-e-TAEG/

11. II video in cui viene mostrato un esempio di utilizzo della legge di capitalizzazione composta con un orizzonte temporale inferiore all'anno è visibile al link: https://www.youtube.com/watch?v=99JnELnCTnw. 
domande di ogni tappa, sia quelle corrette che quelle sbagliate, il numero di tentativi effettuati, i commenti degli studenti al termine del gioco, raccolti tramite un questionario anonimo in cui è stato chiesto un aspetto positivo e uno negativo, e i commenti degli insegnanti che hanno proposto l'iniziativa alle loro classi.

\subsection{Osservazioni su tempi, materiali e spazi}

Per quanto riguarda i tempi di realizzazione di questa proposta, gli insegnanti avevano messo a disposizione due ore di lezione in cui includere tutto l'intervento: la fase di spiegazione iniziale, la formazione delle squadre, la fase di gioco e la premiazione finale. Non sempre questo tempo è stato sufficiente, soprattutto perché la fase di preparazione iniziale in alcuni casi è durata più del previsto e la fase di gioco vera e propria è durata tra i 90 e i 120 minuti. In alcuni casi quindi è stato necessario occupare anche il tempo dell'intervallo o parte delle ore di lezioni successive.

Il gioco è stato proposto in diversi momenti della giornata a seconda delle disponibilità dei docenti: durante le prime ore, nelle ore centrali della mattinata o nelle ultime due ore. Durante due sperimentazioni, al liceo scientifico e presso l'istituto professionale, il gioco si è sovrapposto con l'orario dell'intervallo ma gli studenti sono rimasti coinvolti nelle attività, accettando di non interrompere l'attività e di recuperare la pausa alla fine del gioco. Durante la seconda sperimentazione al liceo scientifico il gioco è stato proposto nelle ultime due ore della mattinata, situazione che abbiamo trovato meno efficace delle altre, vista la preoccupazione degli studenti di dover uscire da scuola per andare a casa e quindi la fretta nel concludere l'ultima tappa.

Quando la sperimentazione ha coinvolto una sola classe, la fase di gioco è stata gestita solo dall'insegnante di matematica della classe e dal ricercatore esterno, mentre con due classi sono stati coinvolti anche altri docenti di supporto all'attività che avevano il compito di trovarsi in una tappa, distribuire il materiale, controllare le risposte e fornire l'indizio successivo. Abbiamo notato che gli studenti sono rimasti colpiti nel vedere anche altri docenti coinvolti, oltre all'insegnante di matematica, e reagivano positivamente ogni volta che incontravano un nuovo insegnante a una tappa.

Per quanto riguarda i materiali a disposizione delle squadre, ogni gruppo poteva usare i propri smartphone per guardare i video, inserire le risposte e utilizzare la calcolatrice. Nonostante prima dell'inizio del gioco avessimo ricordato di portare una penna per scrivere, pochissime squadre hanno autonomamente iniziato a prendere appunti durante la visione dei video, trascrivere le formule o segnarsi i conti da svolgere. Molte squadre hanno utilizzato carta e penna solo dopo il consiglio esplicito da parte dei docenti; I'unico caso in cui non è stato necessario questo consiglio è stata durante la seconda sperimentazione al liceo scientifico quando come gadget di partecipazione è stato fornito a tutti gli studenti un block notes e una penna. In questo caso gli studenti hanno utilizzato spontaneamente il block notes fin da subito per fare calcoli, segnare le risposte, appuntarsi le informazioni più importanti.

Infine, per quanto riguarda gli spazi scelti per ospitare le tappe del gioco, a seconda della disponibilità dell'istituto sono state fatte due scelte diverse: al liceo scientifico tutte le tappe erano nel cortile della scuola; negli altri casi, invece, le tappe erano luoghi sia interni che esterni (aule rimaste libere, biblioteca, bar, aula delle fotocopie ecc.). Ogni volta che una squadra arrivava ad una tappa doveva fermarsi per qualche minuto per guardare i video, leggere i testi e rispondere alle domande. Abbiamo notato che quando le tappe erano ospitate in luoghi più "scolastici", come ad esempio la biblioteca, l'aula di classe, la dispersione e distrazione degli studenti era minore rispetto alle 
Figura 3

Alcune studentesse

mentre cercano le risposte

alla seconda tappa. tappe situate in corridoio o all'esterno, soprattutto nell'affrontare le domande più impegnative. Se all'inizio del gioco trovarsi in cortile a fare matematica è risultato un elemento nuovo e stimolante, successivamente gli studenti sono sembrati più a loro agio nell'affrontare le tappe più difficili in luoghi dove potessero sedersi, appoggiarsi a un tavolo, avere a disposizione spazio per discutere, scrivere e confrontarsi.

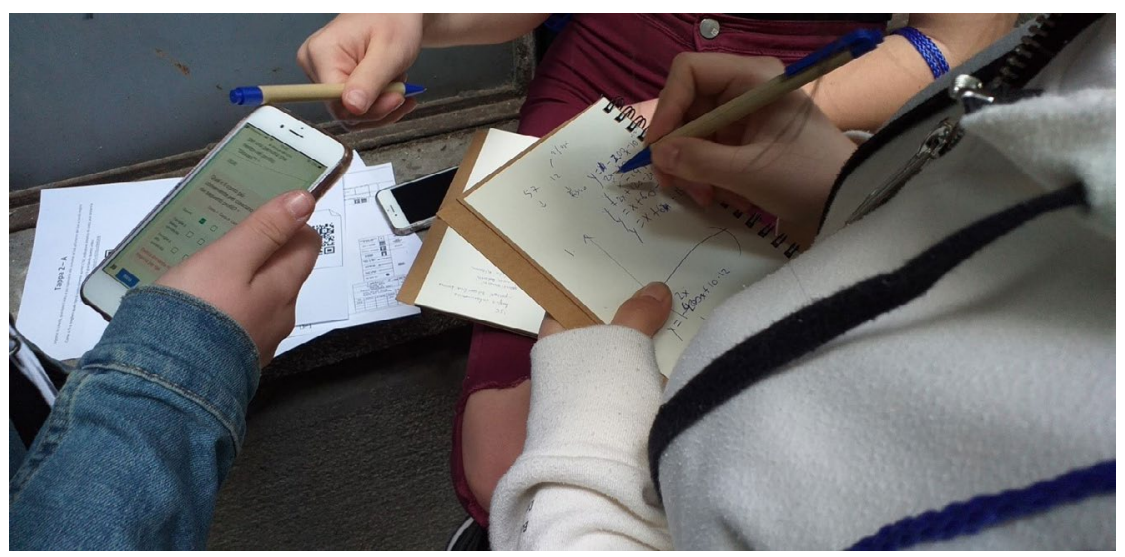

\subsection{Le difficoltà riscontrate}

In tutte le giornate, una delle maggiori difficoltà incontrate dagli studenti è stata di "entrare" nello spirito del gioco. Prima di iniziare il gioco gli studenti avevano ricevuto diverse indicazioni sull'attività che avrebbero realizzato; i docenti avevano anticipato quali sarebbero state le modalità del gioco, chiesto agli alunni di portare con sé gli smartphone con la possibilità di connettersi, ricordato di portare una penna e dei fogli per poter prender appunti in caso di necessità.

Gli studenti sono sembrati attivi e interessati alla proposta, ma spaesati nel momento in cui si sono trovati a confrontarsi con fogli e cellulari, apparentemente disorientati dalla situazione in cui sono stati posti, diversa dalle lezioni a cui sono abituati.

Nonostante la familiarità con gli strumenti tecnologici nella vita quotidiana, spesso i ragazzi non sembrano essere abituati a usare gli smartphone in ambito scolastico. Ad esempio, una cosa che abbiamo osservato durante il gioco è stata che solo alcune squadre sono andate oltre l'indicazione iniziale di avere almeno un cellulare per squadra e hanno iniziato a usare più smartphone con funzioni diverse (per vedere i video, per fare i calcoli, per inserire le risposte ecc.) con il risultato di perdere meno tempo e rendere il gioco più veloce. La maggior parte delle squadre ha utilizzato un solo cellulare, perdendo molto tempo nel continuo aprire nuove finestre e con delle difficoltà di collaborazione e coinvolgimento da parte di tutti i componenti del gruppo. Questo è successo anche nelle scuole in cui era a disposizione la connessione Wi-Fi dell'istituto, per cui gli studenti non dovevano necessariamente utilizzare i loro dati per la connessione. In generale, I'utilizzo del cellulare da parte degli studenti per inserire le risposte non è sembrato così naturale come ci aspettavamo, forse anche a causa dello strumento di Google Forms, poco conosciuto e mai utilizzato prima dai ragazzi. Come abbiamo già osservato, la reticenza nell'utilizzare penne e supporti cartacei, oltre a quelli elettronici, si è manifestata in quasi tutte le sperimentazioni, rendendo difficile recuperare dai video le informazioni essenziali per fornire le risposte, difficoltà derivante anche da un ascolto superficiale dei contenuti.

Nel seguito di questo paragrafo vogliamo analizzare alcune delle domande dei que- 
stionari somministrati agli allievi, fornendo delle possibili interpretazioni degli errori commessi dai ragazzi. Come ci si potrebbe aspettare, le domande chiuse sono risultate mediamente più facili rispetto alle domande aperte univoche, in cui era necessario svolgere un calcolo e inserire la risposta numerica. Per questa analisi abbiamo selezionato per ognuna delle tappe alcune domande significative, dal nostro punto di vista, per i contenuti e per la tipologia di errori presenti. Abbiamo trascurato la prima tappa, "Aula", perché le domande riguardavano argomenti di matematica già noti agli studenti e non introdotti tramite questo gioco.

Per poter interpretare al meglio i numeri che forniremo ricordiamo che nei file delle risposte che abbiamo raccolto da Google Forms sono presenti tutti i tentativi di risposta inseriti dalle squadre che hanno partecipato alla sperimentazione e che ogni squadra aveva a disposizione più di un tentativo. In questi dati notiamo che alcune squadre hanno inserito più di una volta risposte identiche (vedi par. 3.2.2), non sappiamo se inconsapevolmente, per mancanza di dimestichezza con lo strumento, per problemi di connessione, convinzione nella risposta o difficoltà di coordinamento tra i membri del gruppo. Per queste ragioni preferiamo evitare di fornire dati in percentuale del numero di risposte corrette rispetto al numero di risposte inserite, essendo questo dato poco rappresentativo, ma ci concentreremo solo sulle risposte sbagliate e sulle diverse tipologie di errore, fornendo delle possibili interpretazioni. La nostra analisi vuole focalizzarsi quindi più sugli aspetti qualitativi degli errori presenti piuttosto che su quelli quantitativi.

\subsubsection{Il calcolo dell'ISC}

Iniziamo la nostra analisi dalla tappa 2A (vedi Allegato 3) relativa ai costi di gestione dei conti correnti e in particolare dalle due domande aperte che riportiamo inserendo tra parentesi anche la risposta corretta:

1. Qual è l'ISC del conto A per una persona che rientra nel profilo "Giovani"? [10 €]

2. Qual è l'ISC del conto $C$ per una persona che rientra nel profilo "Giovani"? [20 €]

Per rispondere è necessario far riferimento ai dati presenti sulla scheda consegnata in questa tappa. In particolare all'informazione che al profilo giovani corrispondono 164 operazioni annue e ai costi delle due offerte (riportate nella Tabella 2).

Tabella 2

La tabella presente nella tappa "Banca" per

confrontare le condizioni dei conti correnti.

\begin{tabular}{|l|l|l|l|}
\hline & $\begin{array}{l}\text { Operazioni } \\
\text { gratuite annue }\end{array}$ & $\begin{array}{l}\text { Costo operazioni } \\
\text { aggiuntive }\end{array}$ & Canone fisso \\
\hline Conto A & 200 & $2 €$ a operazione & $10 €$ annuale \\
\hline Conto B & 130 & $1 €$ a operazione & $1 €$ a bimestre \\
\hline Conto C & 150 & $1 €$ a operazione & $1,50 €$ a trimestre \\
\hline
\end{tabular}

Nel video segnalato nel materiale di questa tappa viene spiegato che cosa sia l'ISC e mostrato un esempio per il suo calcolo, proprio nel caso di una persona che rientra nel profilo "Giovani" per un conto corrente in cui ci sono $1 €$ di spese fisse mensili, 50 operazioni gratuite all'anno e il costo 0,50 € per ogni operazione aggiuntiva (Figura 4). 
Figura 4

Un fermoimmagine del video della tappa $2 \mathrm{~A}$ in cui viene mostrato come calcolare I'ISC.

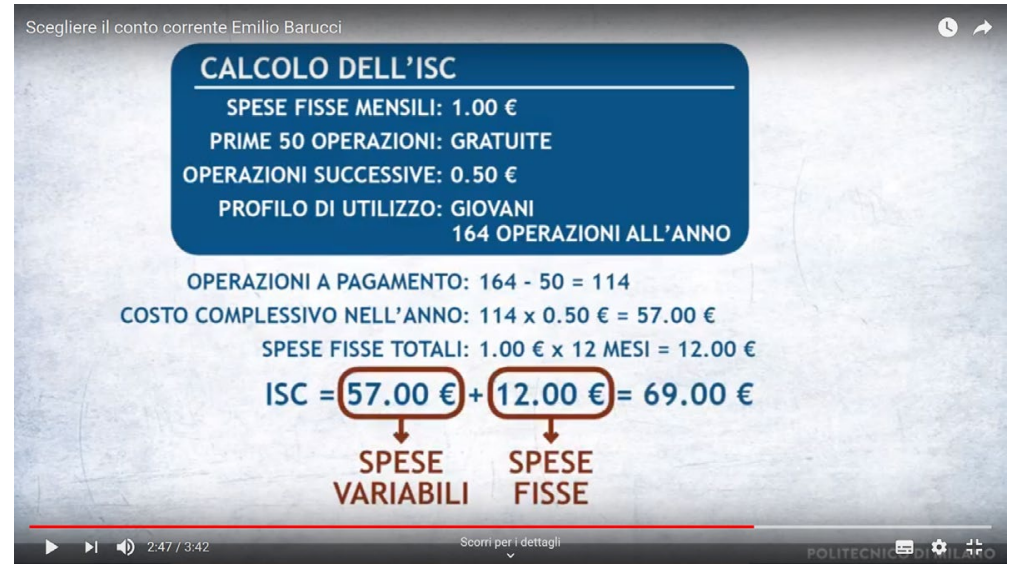

Nel caso del conto A il numero di operazioni gratuite offerte supera il numero di operazioni effettuate, quindi I'ISC del conto corrente corrisponde solo al costo del canone fisso annuale, quindi a $10 €$. Per rispondere alla seconda domanda, relativa al conto $C$, occorre sommare al canone fisso annuale, cioè a $6 €$, le spese variabili (cioè le 14 operazioni a pagamento al costo di $1 €$ l'una), per cui I'ISC è $20 €$. Notiamo che la prima domanda appare essere molto più semplice della seconda, visto che per rispondere non è necessario nessun calcolo ma solo riportare il valore delle spese fisse, già contenuto nella Tabella 2 .

I tentativi di risposta sbagliati, cioè che contengono almeno un errore in una delle due risposte, che abbiamo registrato sono 32, attribuiti a 18 squadre diverse, e sono raccolti nel dettaglio nell'Allegato 7 . In particolare, 16 tentativi di risposta contengono un errore solo nella prima domanda e 7 nella seconda domanda, mentre 9 tentativi contengono errori in entrambe le risposte (Tabella 3).

\begin{tabular}{|l|l|}
\hline Totale di risposte con almeno un errore nelle due domande della tappa 2A & 32 \\
\hline Risposte con errori solo nella domanda 1 & 16 \\
\hline Risposte con errori solo nella domanda 2 & 7 \\
\hline Risposte con errori ad entrambe le domande & 9 \\
\hline
\end{tabular}

Le nove risposte con errori a entrambe le domande sono state fornite da sette squadre nel loro primo tentativo (due squadre hanno commesso due errori anche al secondo tentativo), possiamo supporre che questi ragazzi abbiano prevalentemente avuto delle difficoltà nel capire cosa sia I'ISC o come lo si possa calcolare. Di questi sette, un gruppo ha addirittura inserito delle risposte non numeriche ("Movimenti allo sportello" e "Carte di credito"), mentre un altro gruppo ha inserito due valori negativi (-60 e -16). Visto che I'ISC rappresenta il costo di gestione di un conto corrente, indicare un valore negativo significherebbe che per coprire i costi di un conto corrente non è il cliente a pagare la banca per il servizio offerto, ma viceversa.

Due squadre hanno risposto alla prima domanda inserendo il valore 82 , che si può ottenere dal calcolo

$$
(200-164) \cdot 2+10
$$


Questa risposta può essere quindi ottenuta se al canone fisso (10 €) vengono aggiunte come spesa variabile 36 operazioni (ottenute dalla differenza tra le operazioni gratuite, 200, e le operazioni stimate, 164) al costo di $2 €$ ciascuna. Ipotizziamo che questo errore possa essere dovuto al tentativo di applicare la formula presente nel video indipendentemente dal fatto che nel caso del conto A il numero di operazioni gratuite sia maggiore rispetto a quello delle operazioni effettuate dal profilo giovani. Per fornire la risposta corretta è necessario interpretare il significato della formula presentata nel video (Figura 4) e capire che nel caso in cui il numero di operazioni effettuate sia minore del numero di operazioni gratuite, non sono presenti spese variabili e I'ISC coincide con il canone fisso annuale.

Le difficoltà maggiori riscontrate per il caso del conto A rispetto a quelle riscontrate per il conto C (Tabella 3) fanno ipotizzare che l'interpretazione della formula e la comprensione del suo significato, andando oltre alla sola applicazione con numeri diversi dell'esempio presente nel video, siano tra i punti deboli nella preparazione degli studenti che potrebbero essersi concentrati di più nell'imparare automatismi e procedure standard piuttosto che nella comprensione approfondita dei quesiti.

\subsubsection{Le leggi di capitalizzazione}

La tappa successiva, ovvero la tappa 2B, è incentrata sulle leggi di capitalizzazione (vedi Allegato 4 ) in particolare sono presenti quattro richieste, ovvero calcolare il montante per un investimento di $100 €$ al $4 \%$ per

1. un anno in regime di capitalizzazione composta; [104 €]

2. tre anni in regime di capitalizzazione composta; $[112,49 €]$

3. un anno in regime di capitalizzazione semplice; [104 $€]$

4. tre anni in regime di capitalizzazione semplice. [112 €]

Per rispondere alle domande è sufficiente inserire i numeri forniti dal testo nelle due formule delle leggi di capitalizzazione (vedi par 2.2.2), presentate agli studenti attraverso il video e la scheda consegnati in questa tappa. L'obiettivo di queste domande è mostrare che i due regimi di capitalizzazione sono equivalenti quando l'intervallo di tempo è annuale, mentre il regime di capitalizzazione composta è più vantaggioso per periodi di tempo maggiori all'anno.

I tentativi sbagliati inseriti in questa tappa sono 38, commessi da 20 squadre su 33 (vedi Allegato 7); questo significa che 13 squadre hanno inserito correttamente tutte le risposte al primo tentativo.

Le domande che sono risultate più difficili (come riportato sinteticamente in Tabella 4) sono le domande 2 e 4, quelle relative all'intervallo temporale di 3 anni, ma diversi errori sono stati commessi anche per la domanda 3 , la cui risposta è relativamente semplice visto che si tratta solo di calcolare l'interesse del $4 \%$ e sommarlo al capitale iniziale.

\begin{tabular}{|l|l|}
\hline Totale di risposte con almeno un errore nelle domande della tappa 3 & 38 \\
\hline Risposte con errori nella domanda 1 & 8 \\
\hline Risposte con errori nella domanda 2 & 25 \\
\hline Risposte con errori nella domanda 3 & 18 \\
\hline Risposte con errori nella domanda 4 & 26 \\
\hline
\end{tabular}


Tra gli errori più frequenti (vedi Allegato 7) c'è quello di riportare la stessa risposta della domanda 2 alla domanda 4 : visto che dopo un anno il valore del montante è lo stesso per i due regimi di capitalizzazione, immaginiamo che gli studenti abbiano generalizzato questa regola portando lo stesso valore anche per l'intervallo temporale di tre anni. Questo errore è stato commesso in "entrambe le direzioni": per cinque volte gli studenti hanno risposto 112 a entrambe le domande, mentre in altri otto casi hanno risposto 112,49 .

Tra le altre risposte scorrette inserite, per alcuni casi possiamo immaginare quale sia stata l'origine dell'errore commesso, ad esempio alcune risposte contengono solo I'interesse maturato (come 12 al posto di 112,4 invece di 104), mentre in altre è stato aggiunto due volte il capitale iniziale (ad esempio ottenendo 204 al posto di 104). Alcune risposte presentano degli errori di approssimazione del risultato: le regole fornite all'inizio richiedevano di approssimare i numeri alla seconda cifra decimale, in un paio di casi alla domanda 2 gli studenti hanno risposto con 112,48 o con 112,50 (la risposta corretta è 112,49).

In altri casi, sono presenti altri valori numerici di cui risulta difficile l'interpretazione, ma che mostrano uno scarso controllo sulla verifica della plausibilità del risultato. Ad esempio, alla terza domanda (capitalizzazione semplice dopo 1 anno) ben quattro squadre, in due giornate diverse, hanno risposto $500 €$, immaginando che un tasso di interesse del $4 \%$ per un anno faccia quintuplicare il valore investito. Come nelle risposte fornite per la tappa precedente il processo di controllo sul risultato ottenuto prima di inserirlo e la sua interpretazione a un contesto reale risultano essere dei punti deboli per molti allievi.

Infine, è utile sottolineare come in alcuni casi si sia verificato il fenomeno a cui abbiamo già accennato, per cui alcune squadre hanno inserito più volte le stesse risposte anche dopo aver trovato le risposte corrette e ripetendo gli stessi inserimenti. Ad esempio, per la squadra SPQR (Tabella 5) sono registrati quattro tentativi: uno con le risposte corrette alle 10:13, un tentativo successivo in cui nell'ultima risposta è presente un errore (alle 10:14) e infine due tentativi a distanza di tre minuti con le risposte nuovamente corrette.

Tabella 5

Un esempio delle risposte inserite a pochi minuti di distanza dalla stessa squadra, in grassetto I'unica risposta sbagliata. II numero del tentativo

è stato indicato dagli studenti stessi.

\begin{tabular}{|l|l|l|l|l|l|l|l|}
\hline $\begin{array}{l}\text { Informazioni } \\
\text { cronologiche }\end{array}$ & Punti & $\begin{array}{l}\text { Nome } \\
\text { della } \\
\text { squadra }\end{array}$ & $\begin{array}{l}\text { Numero } \\
\text { del } \\
\text { tentativo }\end{array}$ & $\begin{array}{l}\text { Domanda } \\
1\end{array}$ & $\begin{array}{l}\text { Domanda } \\
2\end{array}$ & $\begin{array}{l}\text { Domanda } \\
3\end{array}$ & $\begin{array}{l}\text { Domanda } \\
4\end{array}$ \\
\hline $\begin{array}{l}4 / 15 / 2019 \\
10: 13: 33\end{array}$ & $4 / 4$ & SPQR & 1 & 104 & 112,49 & 104 & 112 \\
\hline $\begin{array}{l}4 / 15 / 2019 \\
10: 14: 25\end{array}$ & $3 / 4$ & SPQR & 1 & 104 & 112,49 & 104 & 112,49 \\
\hline $\begin{array}{l}4 / 15 / 2019 \\
10: 15: 37\end{array}$ & $4 / 4$ & SPQR & 1 & 104 & 112,49 & 104 & 112 \\
\hline $\begin{array}{l}4 / 15 / 2019 \\
10: 18: 20\end{array}$ & $4 / 4$ & SPQR & 2 & 104 & 112,49 & 104 & 112 \\
\hline
\end{tabular}

\subsubsection{Il confronto tra titoli azionari}

Per quanto riguarda la tappa successiva "Casa" (vedi Allegato 5), le domande sono tutte a risposta chiusa. In particolare, in questa analisi ci concentriamo sulle prime tre domande che riteniamo più significative in quanto erano chieste delle osservazioni a 
partire da un grafico (Figura 2). Le domande poste sono le seguenti:

1. Considerando il grafico dei titoli Appela e Samugu, com'è stato il rendimento dell'azione Samugu comprata nel 2014 e rivenduta nel 2019?
a. Positivo
b. Negativo (risposta corretta)
c. Uguale a zero
d. Non ci sono dati sufficienti per rispondere

2. Considerando il grafico dei titoli Appela e Samugu, qual è stato il rendimento di un'azione Appela che è stata comprata nel 2014 e rivenduta nel 2019?
a. $10 \%$ (risposta corretta)
b. $5 \%$
c. $7 \%$
d. Nessuno di questi
e. Non ci sono dati sufficienti per rispondere

3. Un'azione del titolo Samugu è stata acquistata nel 2017. In quale anno si avrebbe guadagnato rivendendola?
a. Nel 2016
b. Nel 2018 (risposta corretta)
c. Nel 2019
d. Nessuno di questi
e. Non ci sono dati sufficienti per rispondere

Tabella 6

I dati sugli errori commessi nelle prime tre domande della tappa 3 .

In queste tre domande ci sono stati 60 tentativi di risposta con almeno un errore, di cui però solo tre in cui tutte le domande risultano sbagliate, nella Tabella 6 sono riportati i dettagli sulle frequenze di errore nelle tre domande.

\begin{tabular}{|l|l|l|l|}
\hline $\begin{array}{l}\text { Totale di risposte con almeno un } \\
\text { errore nelle tre domande }\end{array}$ & 60 & $\begin{array}{l}\text { Riposte con tutte e tre le } \\
\text { domande sbagliate }\end{array}$ & 3 \\
\hline $\begin{array}{l}\text { Risposte con errore nella } \\
\text { domanda 1 }\end{array}$ & 10 & $\begin{array}{l}\text { Risposte con errori solo nella } \\
\text { domanda 1 e 2 }\end{array}$ & 3 \\
\hline $\begin{array}{l}\text { Risposte con errore nella } \\
\text { domanda 2 }\end{array}$ & 45 & $\begin{array}{l}\text { Risposte con errori solo nella } \\
\text { domanda 1 e 3 }\end{array}$ & 1 \\
\hline $\begin{array}{l}\text { Risposte con errore nella } \\
\text { domanda 3 }\end{array}$ & 28 & $\begin{array}{l}\text { Risposte con errori solo nella } \\
\text { domanda 2 e 3 }\end{array}$ & 13 \\
\hline
\end{tabular}

La maggior parte degli errori commessi si concentrano sulla seconda domanda, in particolare le alternative scorrette (le opzioni b, c, d, e) sono state scelte quasi con la stessa frequenza dagli studenti (Figura 5).

Per rispondere correttamente alla seconda domanda era necessario utilizzare la definizione di rendimento, presente sulla scheda consegnata nella postazione (vedi Allegato 5) e ricavare dal grafico (Figura 2) il prezzo dell'azione nel 2014 (70 €), che corrisponde al prezzo di acquisto, e nel 2017, (77 €), che corrisponde al prezzo di vendita. Per ottenere il rendimento la differenza tra il prezzo di vendita e il prezzo di 
acquisto deve essere divisa per il prezzo di acquisto, la risposta corretta era quindi $10 \%$ ottenuta da

$$
\frac{77-70}{70}=\frac{7}{70}=0,1
$$

La risposta sbagliata più selezionata è stata il 7\%. Tra i ragionamenti che possono aver portato a questa risposta possiamo ipotizzare che questi studenti si siano fermati dopo aver calcolato la differenza tra il prezzo di vendita e di acquisto dell'azione o abbiano avuto delle difficoltà nel trasformare 7/70 in percentuale.

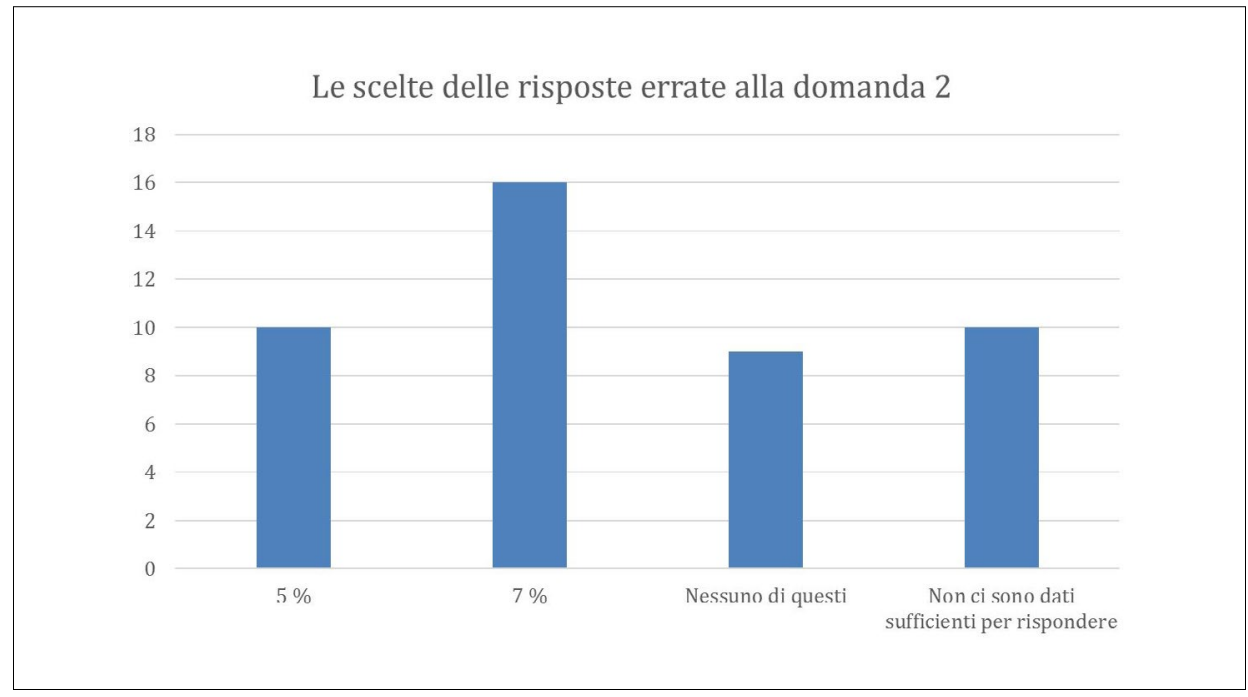

Al di là di quali siano stati gli errori commessi, risulta interessante sottolineare che la domanda 2 che è risultata la più difficile era in effetti quella in cui le richieste erano più complesse: non erano necessari solo la lettura del grafico e una valutazione qualitativa (come nelle domande 1 e 3), ma serviva entrare nello specifico della definizione di rendimento, cogliere le informazioni numeriche dal grafico, svolgere correttamente il conto e trasformare il risultato in un valore in percentuale.

\subsubsection{Tassi di interesse con orizzonti temporali diversi}

Per quanto riguarda l'ultima tappa, vogliamo concentrarci sulle domande conclusive (vedi Allegato 6): due domande aperte in cui viene richiesta l'applicazione della legge di capitalizzazione composta in situazioni in cui il tasso di interesse non è annuale. Le due domande poste sono le seguenti:

1. Chiedi un prestito di $1000 €$, con restituzione ad un anno. Andrea ti può fornire il denaro che ti serve al 4,5\% quadrimestrale, quanto dovrai restituirgli? [1141,17 €]

2. Chiedi un prestito di $1000 €$, con restituzione ad un anno. Maria ti può fornire il denaro che ti serve al $4 \%$ trimestrale, quanto dovrai restituirgli? [1169,86 €]

Queste due domande sono risultate le più difficili di tutto il gioco, forse perché oltre a riguardare un concetto non banale, erano presentate proprio al termine del gioco quando, in tutte le sperimentazioni, abbiamo osservato che tra i ragazzi la stanchezza si è fatta sentire maggiormente e il livello di concentrazione è calato. 
Figura 6

Un fermo immagine del video presente nell'ultima tappa del gioco.
Dal punto di vista matematico, per poter rispondere correttamente a queste domande, è necessario innanzitutto individuare quante volte in un anno verrà applicato il tasso di interesse e utilizzare la capitalizzazione composta per applicare l'interesse non solo al capitale iniziale ma al capitale più l'interesse già sviluppato. Questo significa che la risposta alla prima domanda è data dal risultato di $1000(1+0,045)^{3}$; mentre per la seconda domanda è necessario calcolare $1000(1+0,04)^{4}$. II video mostrato in questa tappa utilizza un esempio molto simile: un tasso di interesse semestrale del $6 \%$ applicato su un capitale iniziale di $100 €$. Inoltre, il video si conclude con una spiegazione in cui la formula necessaria per rispondere a queste due domande viene mostrata esplicitamente e commentata (Figura 6).

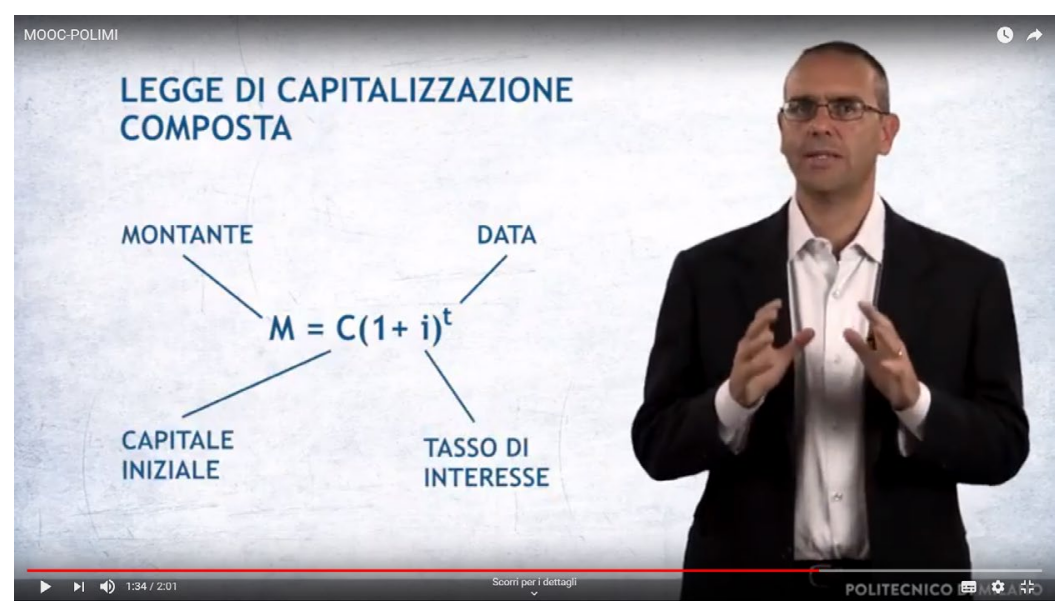

Le risposte sbagliate a queste due domande corrispondono a 62 tentativi registrati (vedi Allegato 7), di cui la maggior parte presenta un errore o un mancato inserimento in entrambe le risposte (Tabella 7).

\begin{tabular}{|l|l|}
\hline Totale tentativi non corretti & 62 \\
\hline Risposte con errore solo domanda 1 & 4 \\
\hline Risposte con errore solo domanda 2 & 6 \\
\hline Risposte con errore entrambe le domande & 31 \\
\hline Risposte vuote a entrambe le domande & 21 \\
\hline
\end{tabular}

La difficoltà di queste due domande potrebbe nascondersi nei diversi passaggi necessari ad ottenere la risposta corretta: individuare la formula della capitalizzazione composta (rivedendola nel video o ricordandola dalla prima tappa), sostituire i numeri con gli opportuni intervalli temporali (in particolare riconoscendo che in un anno sono presenti 3 quadrimestri e 4 trimestri) e infine assicurarsi di inserire correttamente i numeri nella calcolatrice per ottenere il risultato.

Oltre a errori simili a quelli già commentati nel caso della tappa 2B, come utilizzare la capitalizzazione semplice e non quella composta o scrivere solo il valore dell'interesse maturato (vedi par 3.2.2), sono presenti alcune risposte non corrette che sembrano 
essere errori di distrazione (ad esempio risposte in cui è scritto 141,17 al posto di 1141,17 o 1196,86 al posto di 1169,86) oltre a risposte di difficile interpretazione e scarsa plausibilità, come numeri minori della cifra presa in prestito (ad esempio 865 e 840). Per 21 tentativi entrambe le risposte sono state lasciate vuote, alcuni di questi tentativi probabilmente corrispondono alle squadre che hanno rinunciato a completare il gioco, perché il tempo a disposizione era terminato o perché si sono interrotte dopo aver visto che alcune squadre avevano concluso e i vincitori erano già stati individuati; mentre altri tentativi vuoti potrebbero essere ricondotti sempre alle difficoltà tecniche cui abbiamo già accennato in precedenza.

\subsection{I commenti di studenti e insegnanti}

Al termine della sperimentazione agli studenti è stato chiesto di indicare in modo anonimo un aspetto positivo e uno negativo dell'esperienza vissuta. Questi riscontri cartacei sono stati raccolti dalle docenti di classe. Gli insegnanti, invece, nei giorni successivi al gioco hanno avuto l'occasione di confrontarsi tra di loro e con il ricercatore esterno per riflettere sui punti di forza e di criticità della proposta.

Per quanto riguarda il feedback ricevuto dagli studenti, tra i punti positivi segnalati ci sono stati il coinvolgimento dato dall'aspetto competitivo; la collaborazione creatasi tra i membri della squadra per trovare le risposte; I'originalità dei temi trattati e della modalità con cui sono stati presentati; I'impostazione del gioco, in particolare il dover utilizzare il cellulare per guardare i video da cui ricavare le informazioni necessarie per rispondere correttamente alle domande. Tra gli aspetti negativi segnalati ci sono state le difficoltà di alcune tappe; i problemi tecnici riscontrati da alcune squadre con le connessioni dei cellulari; la richiesta di avere più tempo a disposizione per completare il gioco.

Alcuni studenti hanno trovato positivo che le risposte da fornire fossero "calcoli e ragionamenti" e che non fossero necessarie particolari conoscenze su questi temi per affrontare il gioco, visto che tutte le informazioni erano presenti nei video e nei testi forniti all'interno del gioco; mentre altri studenti hanno segnalato questa stessa caratteristica come un punto debole, segnalando delle domande troppo difficili rispetto alla loro preparazione e suggerendo di fare un'introduzione sui temi della matematica finanziaria prima di proporre il gioco. La differenza dei feedback ricevuti rispecchia ovviamente la differenza degli studenti presenti in ogni classe e soprattutto l'eterogeneità delle squadre che si sono formate con il sorteggio casuale. Nella fase di riprogettazione seguita a queste sperimentazioni abbiamo comunque preso la decisione di semplificare il gioco, mantenendo gli stessi contenuti ma diminuendo il numero di domande presentate in ciascuna tappa, in modo che gli studenti potessero focalizzarsi sui concetti principali.

Il riscontro dell'esperienza è stato positivo anche da parte degli insegnanti di matematica coinvolti, in particolare per le due docenti di matematica autrici di questo lavoro che si sono occupate sia della progettazione sia della supervisione del gioco nelle loro classi.

La caccia al tesoro finanziaria è stata una esperienza didattica che ha fatto emergere interesse e coinvolgimento degli alunni per contenuti matematico-finanziari che solitamente non vengono mostrati, in particolare nelle classi del liceo scientifico, percorso scolastico dove non è previsto l'insegnamento di materie economiche e della matematica finanziaria neanche negli anni successivi al biennio. Anche per queste 
ragioni, nel liceo scientifico l'attività è stata ripresa nei giorni successivi per integrarla nella valutazione degli studenti. Agli allievi è stato chiesto di prepararsi riguardando i link delle pagine web già utilizzate durante la gara e a ciascun gruppo è stato chiesto di focalizzarsi su due argomenti a scelta (in modo da rivedere tutti gli argomenti). In questo modo, la discussione aperta dall'insegnante ha sempre trovato interlocutori preparati, mostrando un approccio ancora competitivo nel mostrare la conoscenza acquisita. Le competenze dimostrate in termini di conoscenze, organizzazione dell'esposizione, capacità di creare legami fra gli argomenti e con gli argomenti curricolari, il linguaggio utilizzato, sono stati poi valutati dal docente.

I docenti hanno anche notato che la creazione delle squadre tramite il sorteggio ha rotto le usuali solidarietà e complicità che esistono in classe, favorendone di nuove. Il comportamento generale dei gruppi è stato in parte quello atteso: qualche gruppo con studenti più competenti in matematica ha risolto rapidamente i primi quesiti, per poi talvolta bloccarsi sull'indovinello relativo all'indizio, che richiedeva più intuizione che conoscenze e abilità di tipo scientifico. In alcuni casi, alunni solitamente più deboli in matematica hanno mostrato destrezza e confidenza con calcoli di percentuali e interessi, esprimendo una predisposizione a gestire questioni relative al denaro e vicine alla realtà. L'esperienza si è dimostrata utile dal punto di vista degli insegnanti per conoscere meglio i propri alunni, scoprire talenti non convenzionali che possono essere incoraggiati maggiormente durante il percorso curriculare e che possono contribuire alla valutazione finale.

\section{Conclusioni}

L'obiettivo di questa parte del progetto Edufin@Polimi era quella di realizzare un'attività che permettesse agli studenti delle scuole secondarie di secondo grado di avvicinarsi ai temi della matematica finanziaria con un approccio informale. Si è scelto di realizzare un gioco sotto forma di caccia al tesoro che comprendesse l'utilizzo di strumenti digitali, il movimento in diversi spazi della scuola e la collaborazione con $\mathrm{i}$ propri compagni di classe. Queste scelte sono state fatte per poter rendere i ragazzi attivi nel processo di apprendimento e per indirizzarli verso una maggiore autonomia nella scoperta, acquisizione e approfondimento di nuovi temi. Pensiamo che "il labirinto delle finanze" possa essere una valida soluzione per i docenti interessati a fornire qualche spunto in più ai loro alunni introducendo delle modalità didattiche diverse rispetto a quelle a cui i ragazzi sono solitamente abituati.

Partendo dai consigli dei docenti di far sì che i concetti visti e appresi nel gioco fossero ripercorsi in una fase di consolidamento, abbiamo aggiunto al gioco delle proposte per riprendere gli argomenti in classe: un test a risposta multipla e una serie di problemi, sugli stessi temi presentati nel gioco. II test è stato pensato come occasione di verifica dei contenuti visti durante l'attività; i problemi sono invece problemi aperti, con più soluzioni possibili che possono essere lo spunto per far nascere una discussione in classe e approfondire i concetti presentati più velocemente nel gioco $^{12}$. La creazione di questo materiale è nata soprattutto dal confronto con gli

12. Questo materiale è ancora in fase di revisione e sperimentazione, per cui abbiamo deciso di non allegarlo a questo articolo. I docenti interessati possono però richiederlo a edufin@polimi.it. 
insegnanti coinvolti in queste sperimentazioni che hanno sentito l'esigenza di avere materiale per proseguire l'attività nelle lezioni successive, cercando però anche di mantenere l'obiettivo di incuriosire i ragazzi su questi temi e utilizzare delle metodologie didattiche diverse da quelle tradizionali.

\section{Bibliografia}

Aprea, C., Schultheis, J., \& Stolle, K. (2018). Instructional integration of digital learning games in financial literacy education. In T. A. Lucey \& K. S. Cooter (Eds.), Financial literacy for children and youth (pp. 69-88). New York, Bern, Berlin: Lang.

Bergmann, J., \& Sams, A. (2012). Flip your Classroom: Reach every Student in every Class every Day. New York: Intl Society for Technology.

INVALSI (2017). OCSE PISA 2015: I risultati degli studenti italiani in Financial Literacy. Disponibile in https://www.invalsi.it/invalsi/ri/pisa2015/doc/2017/Rapporto FL PISA2015 24052 017.pdf (consultato il 18.03.2020).

Kaiser, T., \& Menkhoff, L. (2017). Does financial education impact financial literacy and financial behavior, and if so, when?. The World Bank.

Lusardi, A. (2012). Numeracy, financial literacy, and financial decision-making (Working paper number 17821). National Bureau of Economic Research.

Lusardi, A. (2015). Financial literacy skills for the 21st century: Evidence from PISA. Journal of consumer affairs, 49(3), 639-659.

Lusardi, A., Mitchell, O. S., \& Curto, V. (2010). Financial literacy among the young. Journal of consumer affairs, 44(2), 358-380.

OECD (2014). PISA 2012 Results: Students and Money (Volume VI): Financial Literacy Skills for the 21st Century. Parigi: PISA, OECD Publishing.

Autori/Giulia Bernardi ${ }^{\star}$, Elena Brambilla ${ }^{\circ}$ e Paola Landra ${ }^{\star}$

*Dipartimento formazione e apprendimento - SUPSI, Locarno, Svizzera

¿Liceo Scientifico Statale Vittorio Veneto - Milano, Italia

^Istituto Professionale di Stato L. Milani - Meda, Italia

giulia.bernardi@supsi.ch, elena.brambilla@polimi.it, paola.landra@uniupo.it 